\title{
The prospects for polymorphisms shared between species
}

\author{
B. GOLDING \\ Department of Biology, York University, North York, Ontario, Canada, M3J 1P3
}

\begin{abstract}
Recent molecular evidence has shown that many MHC polymorphisms are not only shared between species but are in fact identical at the molecular level. Species that share these polymorphisms may be very distantly related and often diverged millions of generations ago. It is now known that this phenomenon is very unlikely to occur if the alleles are selectively neutral. A large number of other studies suggest, however, that this phenomenon of shared polymorphisms is very common and extends to many other loci beyond just the MHC/HLA complexes. These studies also suggest that some polymorphisms may be older than the MHC/HLA polymorphisms. The maintenance of these polymorphisms via overdominant and frequency-dependent selection is discussed. Strong levels of selection are required for overdominance to maintain shared polymorphisms but most studies of effective population size produce long-term estimates that are very small and would not permit the level of overdominant selection required to maintain these polymorphisms. Frequency-dependent selection can thaintain them for longer with less 'apparent' equilibrium selection and might permit smaller effective sizes. The variance of allele frequencies is suggested to be one way to distinguish between these two selection models.
\end{abstract}

Keywords: effective population size, molecular evolution, shared polymorphisms, trans-species evolution.

\section{Introduction}

The neutral theory of molecular evolution (Kimura, 1983) suggests that most molecular polymorphisms are influenced more by random drift than by selection. Simple expectations can be constructed to predict the pattern of molecular genetic variation that should be observed when selection is absent. Because of the neutral theory of molecular evolution, the presence of many polymorphisms is now explained as a transient event. Gillespie (1984, 1986), however, has demonstrated that the rates of evolution for many genes follow an episodic pattern. He suggests that the best way to explain this pattern of evolution is to invoke selection acting on the different sequence variants.

Polymorphisms that are shared between two or more related species also require a selective explanation for their presence. This phenomenon has been termed 'trans-species evolution' (Arden et al., 1980; Klein, 1980; Arden \& Klein, 1982). These polymorphisms are inferred to have been present in the ancestor of these species and to have survived within both species throughout their divergence. Several recent papers have noted the presence of shared polymorphisms between species and have further demon- strated that these polymorphisms are molecularly identical.

McConnell et al. (1988) found that four of five Mus species shared a polymorphism for a SINE in the middle of an MHC class II gene intron. These species have been distinct for the last 3-4 million years. Sagai et al. (1989) also found a polymorphism for the $\mathrm{H}-2 \mathrm{~K}^{\mathrm{f}}$ antigen shared within the genus Mus. Another MHC polymorphism was detected by Figueroa et al. (1988) which is even more ancient. This consists of a polymorphic deletion of two codons (separated by one undeleted codon) in exon 2 of the mouse $\mathrm{H}-2 \mathrm{~A}_{\mathrm{B}}$ gene. They found that the deletion was variably present or absent in Mus m. domesticus, Mus m. musculus, Mus castaneus, Mus molossinus, Mus spretus, Mus hortulanus, and Rattus norvegicus. Again, the polymorphism extends over an estimated 10 million years since the divergence of the genus Mus from the genus Rattus. This phenomenon has also been detected at the molecular level in primates. Lawlor et al. (1988), Mayer et al. (1988), and Gyllensten \& Erlich (1989) report the presence of different sets of polymorphic alleles in the HLA system which are shared by both humans and chimpanzees. The small amount of sampling that has been done suggests that this pheno- 
menon must be more widespread than currently indicated. According to Parham et al. (1989) more than 50 per cent of 328 HLA substitutions may be shared by humans and chimpanzees.

These reports of molecular MHC/HLA polymorphisms shared between species are not the first suggestion of this phenomenon. It was noted by Balner et al. (1967) that chimpanzees and humans share similar HLA antigens, however, the above papers have demonstrated that these polymorphisms are not only similar but that they are identical at the molecular level. Thus, many other shared polymorphisms (when examined at the molecular level) may also be expected to share a single molecular ancestor.

There have been many reports of such 'similar' polymorphisms shared by several species. Perhaps one of the first cases noted in the literature was by von Dungern \& Hirschfeld (1911) and Landsteiner \& Miller (1925) (cited in Moor-Jankowski et al., 1964). They found that the great apes (excluding gorillas) have an $\mathrm{A}-\mathrm{B}-\mathrm{O}$ blood group polymorphism with reactions that are 'indistinguishable' from those of humans. Hence, at least for the apes, there is a shared polymorphism where three alleles are maintained. In addition to the A-B-O blood group polymorphisms, other classical polymorphisms (such as $\mathrm{M}-\mathrm{N}$ and $\mathrm{Gm}$ ) appear to have close homologues in the great apes (Cavalli-Sforza \& Bodmer, 1971). However, other blood group polymorphisms known to be influenced by strong selection are not shared between species (e.g. Gc - Kitchin \& Bearn, 1965; Rh - Wiener \& MoorJankowski, 1963) so this phenomenon does not encompass all selected polymorphisms.

Fisher et al. (1939) also noted a shared polymorphism that illustrates another important aspect to this phenomenon. They tested the ability of apes to taste phenyl-thiocarbamide (PTC). In humans the frequency of PTC non-tasters is usually within the range of 25-30 per cent. When chimpanzees were tested they found that 26 per cent were incapable of tasting PTC. Thus, they found that not only do the two species share the same polymorphism but, in their sample of 27 chimpanzees, the frequency of the phenotypes are also the same. Chiarelli (1963) also found a PTC polymorphism within the great apes and with similar frequencies to man (chimpanzee 25 per cent, $n=81$; gorilla 22 per cent, $n=18$ ). The polymorphism also exists in orang-utans, gibbons, Old and New World monkeys and in lemurs. The lineages of gibbons and humans diverged approximately 15 million years ago. Fisher et al. (1939) conclude that there must be strong balancing selection for this 'apparently valueless character' to maintain it for this length of time.

Ancient polymorphisms may also be present for geological time periods and can be observed in the fossil record. Owen (1966) examined Limicolaria martensiana fossils estimated to be $8,000-10,000$ years old from the western region of Uganda. He found that these fossils have the same shell colour and patterns as living populations found in the same area. Gould (1988) has also found distinctive patterns of covariance of growth in the snail Cerion agassizi on different islands of the Bahamas. He shows that these patterns have been stable for at least $1.2 \times 10^{5}$ years. Again the suggestion is one of stable polymorphisms that last for millions of generations. Even after these long time periods the frequencies of each phenotype may remain relatively constant.

Many other examples of shared polymorphisms have been noted. In most species, however, these polymorphisms have not been well studied. Given that the MHC/HLA polymorphisms, when examined at the molecular level, were found to be identical, it would not be surprising to find that many of the other known examples turn out to be identically shared polymorphisms. These alleles must therefore have been inherited since the time of speciation.

It has been shown by Takahata \& Nei (1990) that these polymorphisms require selection to explain their existence. The probability of observing this phenomenon for selectively neutral alleles is vanishingly small. Some form of balancing selection is required to maintain the polymorphism for long time periods. Takahata \& Nei (1990) examined overdominant selection (with fitnesses of 1 for heterozygotes and $(1-s)$ for homozygotes) and frequency-dependent selection (with fitnesses of $\left(1-s x_{i}\right)\left(1-s x_{j}\right)$ for genotype $\mathrm{A}_{\mathrm{i}} \mathrm{A}_{\mathrm{j}}$, where $x_{i}$ is the frequency of the $i$ th allele). Both require strong levels of selection to maintain the polymorphisms. Takahata \& Nei (1990) suggest that $N s>1000.0$ is required.

The results of Takahata \& Nei (1990) are confirmed here. It is shown that neutral polymorphisms cannot survive for geological time periods and that to do so requires strong levels of selection. However, the above examples suggest that the phenomenon of trans-species evolution is very widespread, extends to many locus types and to species with even more ancient divergences than those noted for the MHC/HLA polymorphisms. The potential prevalance of this phenomenon requires careful examination of the conditions and prerequisites necessary for shared polymorphisms to evolve. It is confirmed here that strong selection is minimally required and shown that asymmetric allele frequencies require even stronger selection. Furthermore, it is shown that the strength of this selection is so great as to preclude many current estimates of effective population sizes. The possibility of 
frequency-dependent selection and the selective maintenance of these polymorphisms is further discussed.

\section{Methods}

To investigate the long-term action of selection on polymorphisms it is necessary to consider the joint effects of mutation, random drift and selection on two diverging populations. A model is assumed where populations have an effective size of $N$ diploid individuals. Generations are discrete and non-overlapping. Two alleles are possible at a locus and mutation occurs equally between these two alleles at a rate of $\mu$ per gamete, per generation. Selection is included to enforce the maintenance of alleles within the divergent populations. The selection is assumed to be overdominant with fitnesses of 1 for heterozygotes and $1-s_{\mathrm{i}}$ for homozygotes. A transient solution to equations that describe this model can be found if the selection is assumed to be weak. These equations are derived in the Appendix, where it is shown that weak selection cannot maintain shared polymorphisms. These equations cannot be exactly solved with strong selection and so simulations were conducted.

Each population for the simulations had an effective size of $N=100$ diploid individuals (or, in one case, $N=1000$ diploid individuals in order to achieve $4 N s=1000$ ). Allele frequencies were initially set at their equilibrium value. No migration was permitted between the two populations so that species divergence is modelled. Each generation $2 N=200$ random numbers were picked to simulate random genetic drift. If the $i$ th random number is less than the frequency of allele $A$ then the $i$ th gene will be allele $A$, otherwise the $i$ th gene is an $a$. New allele frequencies were then calculated. The allele frequencies were then changed deterministically according to the appropriate mutation rates and selection coefficients. These determined the new allele frequencies and complete a single generation. This process was then repeated for 10,000 generations. At predetermined intervals the populations were examined to determine (i) if both populations were still polymorphic (at the 95 per cent level), and (ii) to determine the allele frequency in one of the populations.

\section{Results}

\section{Neutral alleles}

The expected heterozygosity within one population is calculated in the Appendix for effective population sizes of $N_{\mathrm{e}}=10^{3}, 10^{4}, 10^{5}$ and $10^{6}$. The expected heterozygosity offers a rough measure of the amount of variation maintained within populations. It shows that the heterozygosity in populations that have diverged for $t=10^{5}, 10^{6}, 10^{7}$ and $10^{8}$ generations will have an appreciable value only when $t \leq N_{\mathrm{e}}$. Thus, the probability of picking two different alleles within one population is very small for all values of $t$ when $N_{\mathrm{e}}=10^{3}$. These results agree with the average time to fixation for an allele destined to be fixed at $4 N_{\mathrm{e}}$ generations. This considers only a single population, while for comparison with the observed phenomenon, two populations should be considered. Assuming that the two populations diverged independently of each other, the probability of joint heterozygosity in both populations will be much smaller (the square of the values). Thus it is very unlikely that any neutral polymorphisms could survive for millions of years. This confirms the results of Takahata \& Nei (1990) and permits an explicit statement of the expected heterozygosity for such ancient polymorphisms.

\section{Overdominant alleles}

Polymorphic alleles can be maintained for longer time periods with overdominant selection, however, this increase is very small if $4 N s \leq 0.1\left(s_{1}=s_{2}=s\right)$. This is not a trivial amount of selection. If this selection was of a co-dominant, deleterious form rather than of an overdominant form, then the expected equilibrium frequency of an allele with these selection coefficients $(4 N s=0.1)$ would be $E\left(x_{1}\right)=0.475$ rather than $E\left(x_{1}\right)=0.5$. This level of selection can therefore cause significant allele frequency changes but is not sufficient to prevent random genetic drift from fixing alleles over long time periods.

Simulations to examine larger selection levels give probabilities of joint polymorphism as shown in Table 1. Again, if there is no selection (Table 1a) there is little (less than 1 in 200) chance of retaining a polymorphism in both populations when $t>4 N_{\mathrm{e}}$. When selection is included (Table $1 \mathrm{~b}-\mathrm{d}$ ) the results do not change significantly until the selection becomes very strong. Even with $4 N s=10$ most polymorphisms are lost to random genetic drift. Only with $4 N s=100$ does the selection become sufficiently powerful to overcome this force.

The overdominance in Table 1 is assumed to be symmetrical. To maintain an equilibrium allele frequency of 25 per cent (as in PTC), the overdominance must be asymmetrical. Robertson (1962) has shown that this asymmetry increases the effectiveness of drift and can in fact cause more rapid loss of alleles than is observed for neutral alleles.

Again with asymmetrical overdominance the same qualitative results are found. If there is no selection 
Table 1 Probability of joint polymorphism in two populations that have diverged for $t$ generations with symmetrical overdominance

\begin{tabular}{|c|c|c|c|c|}
\hline & $N_{\mathrm{e}}=10^{6}$ & $N_{\mathrm{e}}=10^{5}$ & $N_{\mathrm{e}}=10^{4}$ & $N_{\mathrm{e}}=10^{3}$ \\
\hline \multicolumn{5}{|c|}{ (a) $4 N s=0.0$} \\
\hline$t=10^{5}$ & $1.000 \pm 0.000$ & $0.595 \pm 0.035$ & $0.0 \pm 0.0$ & $0.0 \pm 0.0$ \\
\hline$t=10^{6}$ & $0.630 \pm 0.034$ & $0.0 \pm 0.0$ & $0.0 \pm 0.0$ & - \\
\hline$t=10^{7}$ & $0.0 \pm 0.0$ & $0.0 \pm 0.0$ & - & - \\
\hline$t=10^{8}$ & $0.0 \pm 0.0$ & - & - & - \\
\hline \multicolumn{5}{|c|}{ (b) $4 N s=1.0$} \\
\hline$t=10^{5}$ & $1.000 \pm 0.000$ & $0.660 \pm 0.034$ & $0.0 \pm 0.0$ & $0.0 \pm 0.0$ \\
\hline$t=10^{6}$ & $0.640 \pm 0.034$ & $0.0 \pm 0.0$ & $0.0 \pm 0.0$ & - \\
\hline$t=10^{7}$ & $0.0 \pm 0.0$ & $0.0 \pm 0.0$ & - & - \\
\hline$t=10^{8}$ & $0.0 \pm 0.0$ & - & - & - \\
\hline \multicolumn{5}{|c|}{ (c) $4 N s=10.0$} \\
\hline$t=10^{5}$ & $1.000 \pm 0.000$ & $0.790 \pm 0.029$ & $0.015 \pm 0.009$ & $0.0 \pm 0.0$ \\
\hline$t=10^{6}$ & $0.790 \pm 0.029$ & $0.005 \pm 0.005$ & $0.0 \pm 0.0$ & - \\
\hline$t=10^{7}$ & $0.005 \pm 0.005$ & $0.0 \pm 0.0$ & - & - \\
\hline$t=10^{8}$ & $0.0 \pm 0.0$ & - & - & - \\
\hline \multicolumn{5}{|c|}{ (d) $4 N s=100.0$} \\
\hline$t=10^{5}$ & $1.000 \pm 0.000$ & $1.000 \pm 0.000$ & $1.000 \pm 0.000$ & $0.990 \pm 0.007$ \\
\hline$t=10^{6}$ & $1.000 \pm 0.000$ & $1.000 \pm 0.000$ & $0.995 \pm 0.005$ & - \\
\hline$t=10^{7}$ & $1.000 \pm 0.000$ & $0.995 \pm 0.005$ & - & - \\
\hline$t=10^{8}$ & $1.000 \pm 0.000$ & - & - & - \\
\hline
\end{tabular}

This table gives the probability of a simultaneous polymorphism in two different populations for selected alleles (overdominant selection with $4 N s$ as indicated). The simulation was repeated 200 times to calculate the mean and its standard error. The simulation was done with an effective population size of $N=100$ diploid individuals $(2 N=200)$. The mutation rate was adjusted to an amount equivalent to $\mu=10^{-9}$. Dashed entries indicate samples not obtained due to the excessive computer time required.

there is little (less than 1 in 200) chance of retaining a polymorphism in both populations when $t>4 N_{\mathrm{e}}$. When selection is included the results are similar to those in Table 1 except that the ability of selection to maintain the polymorphism has become much weaker The probability of joint polymorphism in two populations is shown in Table 2 for alleles with an equilibrium frequency $x_{1}=0.75$ rather than $x_{1}=0.5$ (fitnesses of $1-s_{1}, 1$, and $\left.1-s_{2}\right)$ and with $4 N\left(s_{1}+s_{2}\right)=100$ or 1000. Even with $4 N\left(s_{1}+s_{2}\right)=100$ most polymorphisms are lost to random genetic drift. Only when selection becomes much stronger is the survival of the polymorphism assured (a $4 N s=1000$ as suggested by Takahata \& Nei, 1990).

\section{Extreme frequency-dependent selection}

It is possible that frequency-dependent selection may be important in the maintenance of the MHC/HLA polymorphisms (and potentially the other poly- morphisms as well). A model of frequency dependence was studied by Takahata \& Nei $(1990)$ with fitnesses of the $A_{\mathrm{i}} A_{\mathrm{j}}$ genotype as $\left(1-s x_{\mathrm{i}}\right)\left(1-s x_{\mathrm{j}}\right)$ and where $x_{\mathrm{i}}$ is the frequency of the $i$ th allele. They showed that this model of frequency dependence will give exactly the same results as overdominant selection as both models cause identical changes in allele frequencies $\left(\Delta x_{i}\right.$ 's). There are many ways that selection can have a frequency-dependent form. Some of these may be less powerful at maintaining a polymorphism than overdominance and others may be more powerful. To determine the effects of other forms of frequency dependence a model of selection was chosen that had a large effect when alleles became rare. This is a model with fitnesses $1+s_{1} /\left(1-x_{2}^{2}\right), 1+s_{1} /\left(1-x_{2}^{2}\right), 1+s_{2} / x_{2}^{2}$ for genotypes $A_{1} A_{1}, A_{1} A_{2}$, and $A_{2} A_{2}$ respectively, where $x_{2}$ is the frequency of $A_{2}$ (Hedrick, 1983). The results for this model are shown in Table 3. To achieve an equilibrium frequency of 0.5 it is necessary to have $s_{1}=3 s_{2}$. The values in Table 3 are with $4 N\left(s_{1}+s_{2}\right)=0.1$, 
Table 2 Probability of joint polymorphism in two populations that have diverged for $t$ generations with asymmetrical overdominance

\begin{tabular}{|c|c|c|c|c|}
\hline & $N_{\mathrm{e}}=10^{6}$ & $N_{\mathrm{e}}=10^{5}$ & $N_{\mathrm{e}}=10^{4}$ & $N_{\mathrm{e}}=10^{3}$ \\
\hline \multicolumn{5}{|c|}{ (a) $4 N\left(s_{1}+s_{2}\right)=100.0$} \\
\hline$t=10^{5}$ & $1.000 \pm 0.000$ & $0.815 \pm 0.028$ & $0.060 \pm 0.017$ & $0.0 \pm 0.0$ \\
\hline$t=10^{6}$ & $0.810 \pm 0.028$ & $0.065 \pm 0.017$ & $0.0 \pm 0.0$ & - \\
\hline$t=10^{7}$ & $0.095 \pm 0.021$ & $0.0 \pm 0.0$ & - & - \\
\hline$t=10^{8}$ & $0.005 \pm 0.005$ & - & - & - \\
\hline \multicolumn{5}{|c|}{ (b) $4 N\left(s_{1}+s_{2}\right)=1000.0$} \\
\hline$t=10^{5}$ & $1.000 \pm 0.000$ & $1.000 \pm 0.000$ & $1.000 \pm 0.000$ & - \\
\hline$t=10^{6}$ & $1.000 \pm 0.000$ & $1.000 \pm 0.000$ & - & - \\
\hline$t=10^{7}$ & $1.000 \pm 0.000$ & - & - & - \\
\hline$t=10^{8}$ & - & - & - & - \\
\hline
\end{tabular}

This table gives the probability of a simultaneous polymorphism in two different populations for selected alleles with asymmetrical overdominant selection (with $4 \mathrm{Ns}$ as indicated). The simulation was repeated 200 times to calculate the mean and its standard error.The simulation was done with an effective population size of $N=100$ diploid individuals $[2 N=200$, (a) or with $N=1000$ (b)]. The mutation rate was adjusted to the amount equivalent to $\mu=10^{-9}$. Dashed entries indicate samples not obtained due to the excessive computer time required.

Table 3 Probability of joint polymorphism in two populations that have diverged for $t$ generations with symmetrical frequency dependent selection

\begin{tabular}{|c|c|c|c|c|}
\hline & $N_{\mathrm{e}}=10^{6}$ & $N_{\mathrm{e}}=10^{5}$ & $N_{\mathrm{e}}=10^{4}$ & $N_{\mathrm{e}}=10^{3}$ \\
\hline \multicolumn{5}{|c|}{ (a) $4 N\left(s_{1}+s_{2}\right)=0.1$} \\
\hline$t=10^{5}$ & $1.000 \pm 0.000$ & $0.625 \pm 0.034$ & $0.010 \pm 0.007$ & $0.0 \pm 0.0$ \\
\hline$t=10^{6}$ & $0.665 \pm 0.033$ & $0.005 \pm 0.005$ & $0.005 \pm 0.005$ & - \\
\hline$t=10^{7}$ & $0.025 \pm 0.011$ & $0.010 \pm 0.007$ & - & - \\
\hline$t=10^{8}$ & $0.0 \pm 0.0$ & - & - & - \\
\hline \multicolumn{4}{|c|}{ (b) $4 N\left(s_{1}+s_{2}\right)=1.0$} & $0.330 \pm 0.033$ \\
\hline$t=10^{6}$ & $0.660 \pm 0.034$ & $0.295 \pm 0.032$ & $0.240 \pm 0.030$ & - \\
\hline$t=10^{7}$ & $0.325 \pm 0.033$ & $0.340 \pm 0.034$ & - & - \\
\hline$t=10^{8}$ & $0.335 \pm 0.033$ & - & - & - \\
\hline \multicolumn{5}{|c|}{ (c) $4 N\left(s_{1}+s_{2}\right)=10.0$} \\
\hline $\begin{array}{l}t=10^{5} \\
t=10^{6}\end{array}$ & $1.000 \pm 0.000$ & $1.000 \pm 0.000$ & $0.990 \pm 0.007$ & $0.995 \pm 0.005$ \\
\hline $\begin{array}{l}t=10^{6} \\
t=10^{7}\end{array}$ & $0.995 \pm 0.005$ & $0.990 \pm 0.007$ & $0.985 \pm 0.009$ & - \\
\hline $\begin{array}{l}t=10^{7} \\
t=10^{8}\end{array}$ & $1.000 \pm 0.000$ & $1.000 \pm 0.000$ & - & - \\
\hline$t=10^{8}$ & $0.990 \pm 0.007$ & - & 一 & - \\
\hline \multicolumn{5}{|c|}{ (d) $4 N\left(s_{1}+s_{2}\right)=100.0$} \\
\hline $\begin{array}{l}t=10^{5} \\
t=10^{6}\end{array}$ & $1.000 \pm 0.000$ & $\begin{array}{l}1.000 \pm 0.000 \\
1.000 \pm 0.000\end{array}$ & $\begin{array}{l}1.000 \pm 0.000 \\
1.000 \pm 0.000\end{array}$ & - \\
\hline$t=10^{6}$ & $1.000 \pm 0.000$ & $\begin{array}{l}1.000 \pm 0.000 \\
1.000 \pm 0.000\end{array}$ & - & - \\
\hline$t=10^{7}$ & $1.000 \pm 0.000$ & $\begin{array}{l}1.000 \pm 0.000 \\
-\end{array}$ & - & - \\
\hline$t=10^{8}$ & $1.000 \pm 0.000$ & - & & \\
\hline
\end{tabular}

This table gives the probability of a simultaneous polymorphism in two different populations for frequency-dependent selection. The simulation was repeated 200 times to calculate the mean and its standard error. The simulation was done with an effective population size of $N=100$ diploid individuals $(2 N=200)$. The mutation rate was adjusted to an amount equivalent to $\mu=10^{-9}$. Dashed entries indicate samples not obtained due to the excessive computer time required. 
1, 10 and 100. This form of frequency-dependent selection can maintain the polymorphism without as large levels of selection. Nor is this ability as quickly impaired by asymmetric selection. If $s_{1}=15 s_{2}$, so that the equilibrium frequency of $A_{1}=0.75$, then a joint polymorphism can still be maintained in roughly 50 per cent of the simulations with $4 N\left(s_{1}+s_{2}\right)=10$ (Table 4).

It is not surprising that this form of frequencydependent selection can maintain polymorphisms more easily than overdominant selection as the frequency-dependent selection becomes very strong as alleles become rare. It might be difficult to discern differences between the two forms of selection. One feature that may be useful is the variance of allele frequencies within populations. In all cases the selection needs to be very strong and there is generally little stochastic influence of finite population sizes. Thus when alleles are maintained over geological time periods the selection must act in essentially a deterministic fashion and there will be small variances in allele frequencies. This is illustrated by Fisher et al.'s (1939) observations on PTC. The allele frequency and its standard deviation within segregating populations is shown in Table 5 for $N_{\mathrm{e}}=10^{4}$. The allele frequencies are sampled from one population at the indicated time and are considered only if the population is still segregating. Hence the standard deviation measures the variation expected between conceptually replicate populations that still retain the polymorphism. Because frequency-dependent selection does not gain strength until alleles become rare it can maintain alleles for longer time periods and with much greater variance in allele frequencies. For example, the standard deviation for symmetric overdominance with $4 N s=100$ is half that for frequency-dependent selection with $4 N s=10$, yet both can maintain the polymorphism. Obviously the variance will decrease with ever increasing levels of selection but the maintenance of the polymorphism can be assured and greater variances achieved by frequencydependent selection than by overdominance.

\section{Other frequency-dependent models}

It has been shown by Denniston \& Crow (1990) that different fitness models can yield the same allele frequency dynamics. The alternate fitness models are often frequency-dependent. Thus Takahata \& Nei $(1990)$ note that fitnesses $\left(1-s x_{\mathrm{i}}\right)\left(1-s x_{\mathrm{j}}\right)$ for genotype $A_{\mathrm{i}} A_{\mathrm{j}}$, will give equivalent results to a model with overdominant fitnesses.

As illustrated by the previous frequency-dependent model, however, not all fitness models give identical dynamics. In fact, there are many ways that fitness can

Table 4 Probability of joint polymorphism in two populations that have diverged for $t$ generations with asymmetrical frequency-dependent selection

\begin{tabular}{|c|c|c|c|c|}
\hline & $N_{\mathrm{e}}=10^{6}$ & $N_{\mathrm{e}}=10^{5}$ & $N_{\mathrm{e}}=10^{4}$ & $N_{\mathrm{e}}=10^{3}$ \\
\hline \multicolumn{5}{|c|}{ (a) $4 N\left(s_{1}+s_{2}\right)=1.0$} \\
\hline$t=10^{5}$ & $1.000 \pm 0.000$ & $0.660 \pm 0.034$ & $0.155 \pm 0.026$ & $0.045 \pm 0.015$ \\
\hline$t=10^{6}$ & $0.675 \pm 0.033$ & $0.095 \pm 0.021$ & $0.055 \pm 0.016$ & - \\
\hline$t=10^{7}$ & $0.100 \pm 0.021$ & $0.095 \pm 0.021$ & - & - \\
\hline$t=10^{8}$ & $0.070 \pm 0.018$ & - & - & - \\
\hline \multicolumn{5}{|c|}{ (b) $4 N\left(s_{1}+s_{2}\right)=10.0$} \\
\hline$t=10^{5}$ & $1.000 \pm 0.000$ & $0.815 \pm 0.028$ & $0.565 \pm 0.035$ & $0.585 \pm 0.035$ \\
\hline$t=10^{6}$ & $0.795 \pm 0.029$ & $0.555 \pm 0.035$ & $0.550 \pm 0.035$ & - \\
\hline$t=10^{7}$ & $0.615 \pm 0.034$ & $0.515 \pm 0.035$ & - & - \\
\hline$t=10^{8}$ & $0.585 \pm 0.035$ & - & - & - \\
\hline \multicolumn{5}{|c|}{ (c) $4 N\left(s_{1}+s_{2}\right)=100.0$} \\
\hline$t=10^{5}$ & $1.000 \pm 0.000$ & $1.000 \pm 0.000$ & $0.990 \pm 0.007$ & $1.000 \pm 0.000$ \\
\hline$t=10^{6}$ & $1.000 \pm 0.000$ & $1.000 \pm 0.000$ & $1.000 \pm 0.000$ & - \\
\hline $\begin{array}{l}t=10^{\prime} \\
t=10^{8}\end{array}$ & $\begin{array}{l}0.985 \pm 0.009 \\
1.000+0.000\end{array}$ & $1.000 \pm 0.000$ & - & - \\
\hline & & - & - & - \\
\hline
\end{tabular}

This table gives the probability of a simultaneous polymorphism in two different populations for frequency-dependent selection. The simulation was repeated 200 times to calculate the mean and its standard error. The simulation was done with an effective population size of $N=100$ diploid individuals $(2 N=200)$. The mutation rate was adjusted to an amount equivalent to $\mu=10^{-9}$. Dashed entries indicate samples not obtained due to the excessive computer time required. 
Table 5 Allele frequencies and their standard deviation sampled from one population with balancing selection

\begin{tabular}{|c|c|c|c|c|}
\hline & \multicolumn{2}{|c|}{ Overdominant selection } & \multicolumn{2}{|c|}{ Frequency-dependent selection } \\
\hline & Symmetric & Asymmetric & Symmetric & Asymmetric \\
\hline \multicolumn{5}{|c|}{ (a) $4 N\left(s_{1}+s_{2}\right)=1.0$} \\
\hline$t=10^{5}$ & $\begin{array}{c}0.695 \pm 0.106 \\
(2)\end{array}$ & $\begin{array}{c}0.307 \pm 0.246 \\
(3)\end{array}$ & $\begin{array}{c}0.491 \pm 0.275 \\
(112)\end{array}$ & $\begin{array}{c}0.473 \pm 0.301 \\
(72)\end{array}$ \\
\hline$t=10^{6}$ & $\begin{array}{c}0.0 \pm 0.0 \\
(0)\end{array}$ & $\begin{array}{c}0.0 \pm 0.0 \\
(0)\end{array}$ & $\begin{array}{c}0.497 \pm 0.301 \\
(99)\end{array}$ & $\begin{array}{c}0.574 \pm 0318 \\
(39)\end{array}$ \\
\hline \multicolumn{5}{|c|}{ (b) $4 N\left(s_{1}+s_{2}\right)=10.0$} \\
\hline$t=10^{5}$ & $\begin{array}{c}0.408 \pm 0.276 \\
(15)\end{array}$ & $\begin{array}{c}0.721 \pm 0.246 \\
(4)\end{array}$ & $\begin{array}{c}0.484 \pm 0.177 \\
(200)\end{array}$ & $\begin{array}{c}0.748 \pm 0.163 \\
(139)\end{array}$ \\
\hline$t=10^{6}$ & $\begin{array}{c}0.0 \pm 0.0 \\
(0)\end{array}$ & $\begin{array}{c}0.0 \pm 0.0 \\
(0)\end{array}$ & $\begin{array}{c}0.503 \pm 0.194 \\
(198)\end{array}$ & $\begin{array}{c}0.751 \pm 0.162 \\
(147)\end{array}$ \\
\hline \multicolumn{5}{|c|}{ (c) $4 N\left(s_{1}+s_{2}\right)=100.0$} \\
\hline$t=10^{5}$ & $\begin{array}{c}0.497 \pm 0.110 \\
(200)\end{array}$ & $\begin{array}{c}0.753 \pm 0.106 \\
(39)\end{array}$ & $\begin{array}{c}0.500 \pm 0.068 \\
(200)\end{array}$ & $\begin{array}{c}0.759 \pm 0.075 \\
(199)\end{array}$ \\
\hline$t=10^{6}$ & $\begin{array}{c}0.498 \pm 0.094 \\
(199)\end{array}$ & $\begin{array}{c}0.0 \pm 0.0 \\
(0)\end{array}$ & $\begin{array}{c}0.491 \pm 0.067 \\
(200)\end{array}$ & $\begin{array}{c}0.753 \pm 0.083 \\
(200)\end{array}$ \\
\hline
\end{tabular}

In all cases $N_{\mathrm{e}}=10^{4}$. The allele frequency and standard deviation are calculated from one of the two populations simulated. Only segregating populations are considered and the number still segregating (out of 200) is shown in parenthesis under the allele frequency. The mutation rate was adusted to an amount equivalent to $\mu=10^{-9}$.

Table 6 Models of frequency-dependent fitnesses

\begin{tabular}{llll}
\hline $\begin{array}{l}\text { Model } \\
\text { number }\end{array}$ & $\mathrm{A}_{1} \mathrm{~A}_{1}$ & $\mathrm{~A}_{1} \mathrm{~A}_{2}$ & $\mathrm{~A}_{2} \mathrm{~A}_{2}$ \\
\hline 0 & $1-s_{1}$ & 1 & $1-s_{2}$ \\
I & $1+s_{1} /\left(1-x_{2}^{2}\right)$ & $1+s_{1} /\left(1-x_{2}^{2}\right)$ & $1+s_{2} / x_{2}^{2}$ \\
II & $1+s_{1}\left(1-2 x_{1}\right)$ & 1 & $1-s_{2}\left(1-2 x_{1}\right)$ \\
III & $1+s_{1}\left(1-x_{2}^{2}\right)$ & $1+s_{1}\left(1-x_{2}^{2}\right)$ & $1+s_{2} x_{2}^{2}$ \\
IV & $1-s_{1} x_{1}^{2}$ & $1-2 s_{2} x_{1} x_{2}$ & $1-s_{1} x_{2}^{2}$ \\
V & $1-s_{1}+s_{2} x_{2}$ & 1 & $1+s_{1}-s_{2} x_{2}$ \\
\hline
\end{tabular}

be made frequency-dependent. To examine some of these other models, further simulations were made. These used four frequency-dependent fitness models that have been widely studied (Wright, 1969; Jacquard, 1974; Spiess, 1977; Ewens, 1979). These models, including the previous models, are shown in Table 6 . Each of these fitness models has a selectively maintained polymorphic equilibrium.

For each model the amount of selection that was required to maintain a shared polymorphism 5 per cent of the time after $t=10^{6}$ generations $\left(N_{\mathrm{e}}=10^{4}\right)$ was determined by simulation. This amount of selection marks the border between maintaining and losing the

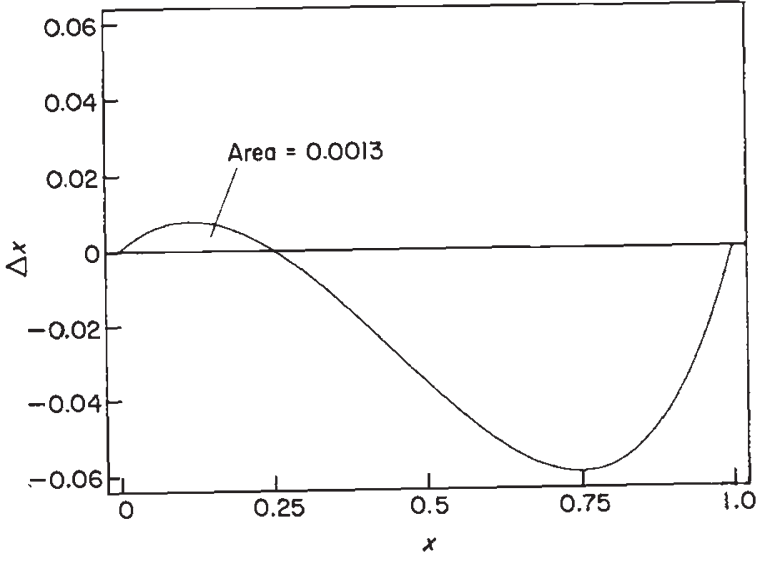

Fig. 1 The change in allele frequency $(x)$ as a function of $x$ with overdominant selection. Fitnesses are $4 N_{\mathrm{e}} s_{1}=150$, $4 N_{\mathrm{e}} s_{2}=50$, with $N=100$.

polymorphism; a little less selection and the polymorphism is lost, a little more and the polymorphism has a good chance of being retained in both populations. For asymmetric overdominance $\left(\hat{x}_{1}=0.25\right)$ sufficient selection occurs when $4 N_{\mathrm{e}} s_{1}=150,4 N_{\mathrm{e}} s_{2}=50$. The relative amount of selection can be measured using a plot of the change in $x$ versus $x$ (Fig. 1) assum- 
ing an infinite population size. The selective pressure that random drift must overcome to fix an allele is measured by the area under the curve of $\Delta x$; note that the area under the curve from $x_{1}=0.0$ to $x_{1}=0.25$ is 0.0013 .

For each of the other models in Table 6, simulations were done to determine the selection coefficients necessary to retain the polymorphism 5 per cent of the time. For each model an equilibrium of $\hat{x}_{1}=0.25$ was chosen but for model III two different equilibria with different properties are possible. These are designated as models IIIa and IIIb with an equilibrium of $\hat{x}_{1}=0.75$ and an equilibrium of $\hat{x}_{1}=0.25$, respectively. The required selection coefficients are

$\begin{array}{lll}\text { No. I } & 4 N_{\mathrm{e}} s_{1}=5, & 4 N_{\mathrm{e}} s_{2}=6 ; \\ \text { No. II } & 4 N_{\mathrm{e}} s_{1}=-380, & 4 N_{\mathrm{e}} s_{2}=127 \\ \text { No. IIIa } & 4 N_{\mathrm{e}} s_{1}=-25, & 4 N_{\mathrm{e}} s_{2}=-380 \\ \text { No. IIIb } & 4 N_{\mathrm{e}} s_{1}=-73, & 4 N_{\mathrm{e}} s_{2}=-57 \\ \text { No. IV } & 4 N_{\mathrm{e}} s_{1}=141, & 4 N_{\mathrm{e}} s_{2}=65 \\ \text { No. V } & 4 N_{\mathrm{e}} s_{1}=151, & 4 N_{\mathrm{e}} s_{2}=202\end{array}$

The maximum selection coefficients examined were $s_{\mathrm{i}}= \pm 0.95$, and yet at this level (with $N=100$ in the simulations), neither model II nor model IIIa could maintain the polymorphism. A plot of $\Delta x$ versus $x$ for each of these models is shown in Fig. 2. This plot explains some of the behaviours of these fitness models. Model I has quite unusual dynamics because the fitness of genotypes tends to infinity as they become rare. Hence $\Delta x$ is large even when $x$ is small and this is quite a strong force to retain alleles in the population. Selection will drive fixation with $x_{1}=1$ in model II whenever $x_{1}>0.5$, and hence the inability to maintain polymorphisms. Model IIIa again has unusual dynamics and will not maintain polymorphisms even with much larger selection coefficients and population sizes. Models IIIb, IV and V all maintain the polymorphism with these selection coefficients. Because of the multiple stable equilibria, model IV has an even larger variance in allele frequency than does model I.

Each of these different frequency-dependent fitness models have differing abilities to maintain a shared polymorphism. Some models cannot maintain the polymorphism as easily as does simple overdominance. Weak frequency dependence, as in model II, cannot maintain the polymorphism at all or weak dependence, as in model $\mathrm{V}$, can only maintain the polymorphism with large selection coefficients $\left[4 N_{\mathrm{e}}\left(s_{1}+s_{2}\right)=353\right]$. To maintain a shared polymorphism the frequency dependence must be relatively strong. This strong frequency dependence permits much smaller selection coefficients [as in model I; $4 N_{\mathrm{e}}\left(s_{1}+s_{2}\right)=11$ ] while still retaining the polymorphism.

\section{Discussion}

The phenomenon of shared polymorphisms implies that these alleles are extremely stable and have survived for millions of years. Because these polymorphisms share identical alleles at the molecular level, they cannot be explained by a simple neutral model (Takahata \& Nei, 1990). Most neutral polymorphisms are retained for only an average of $4 N_{\mathrm{e}}$ generations.

More surprisingly, even with moderate selection actively maintaining the polymorphism, random genetic drift will still eventually eliminate the polymorphism in one of the species. This is, in part, due to the long time periods that characteristically separate two species. Only when selection becomes very strong is a shared polymorphism likely.

The strength of the selection implied is quite dramatic and yet many of these polymorphisms are of unknown selective value to their hosts. The MHC and HLA polymorphisms are strongly implicated in disease resistance and hence speculation is that they may periodically be under strong selection. Actual observations and measurements of these selective events, however, is still largely lacking. Evidence for other loci is more equivocal. Even for the A-B-O polymorphism Cavalli-Sforza \& Bodmer (1971) state that 'in spite of the enormous amount of published work aimed at uncovering the selective forces associated with the A-B-O polymorphism, no real understanding of such forces has yet emerged' (page 214). Similarly Fisher et al. (1939) noted that the PTC polymorphism was an 'apparently valueless character'. The selection pressures on these polymorphisms should be much more apparent than current observations would suggest.

The results presented are conservative in several ways. All initial conditions were with the two alleles at their equilibrium frequency. At this frequency the loss of variation due to genetic drift will be maximally postponed. With more than two alleles and with unequal allele frequencies, random drift will more quickly fix one of the species. Mutations in both directions between alleles were permitted yet many of the molecular alleles are complicated changes that are unlikely to arise more than once by mutation and less likely to revert. The small mutation pressure acts to maintain the polymorphism longer than it would be in nature. No population bottlenecks have been considered associated with speciation, however many views of speciation would suggest that a severe reduction in numbers is associated with speciation. This would again increase the rate of fixation for species and forestall the active maintenance of polymorphisms due to selection.

These results have implications for the range of per- 

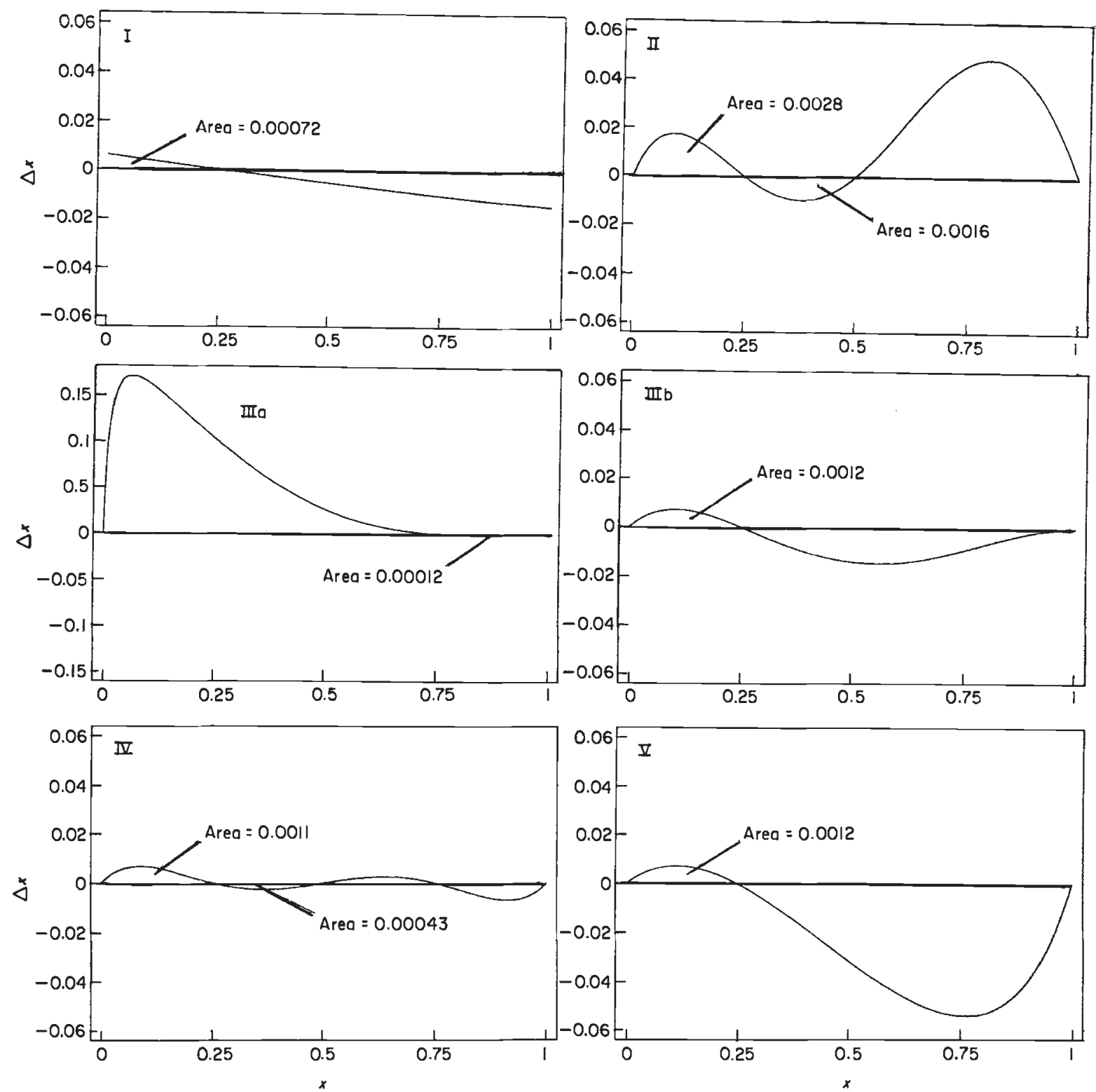

Fig. 2 The change in allele frequency $(x)$ as a function of $x$ with frequency-dependent selection. The type of frequency dependence is shown in Table 6. Fitnesses are No. I $4 N_{\mathrm{e}} s_{1}=5,4 N_{\mathrm{e}} s_{2}=6$; No. II $4 N_{\mathrm{e}} s_{1}=-380,4 N_{\mathrm{e}} s_{2}=127$; No. III $4 N_{\mathrm{e}} s_{1}=-25$, $4 N_{\mathrm{e}} s_{2}=-380$; No. IIIb $4 N_{\mathrm{e}} s_{1}=-73,4 N_{\mathrm{e}} s_{2}=-57$; No. IV $4 N_{\mathrm{e}} s_{1}=141,4 N_{\mathrm{e}} s_{2}=65$; No. V $4 N_{\mathrm{e}} s_{1}=151,4 N_{\mathrm{e}} s_{2}=202$, with $N=100$. Models II and IIIa are unable to maintain a shared polymorphism (note that model IIIa has a different scale).

missable effective populations sizes. A collection of studies that have estimated the effective size of populations using genetic data is given in Table 7. For comparison, an estimate of the effective population size of primitive man using extensive short-term demographic (but no genetic) information found $N_{\mathrm{e}}=650$ (Wood, 1987). The majority of these studies indicate an $N_{\mathrm{e}}$ less than a few thousand. The notable exception being the studies by Avise et al. (1988). In their case, species were chosen based on their large census size and known high rates of historical gene flow. Even in this case, however, the effective sizes are up to 900 -fold smaller than the census size. On the other hand, Nei \& Graur (1984) used current levels of heterozygosity, estimates of generation length and estimates of neutral mutation rates to infer the effective population size of a wide range of species. Their estimates fall mostly in the range of $10^{4}-10^{6}$ (with man at the low end of this scale and fruit flies at the high end). Hence, they note that an $N s$ of 1000 does not require actual selection coeffi- 
Table 7 Estimates of effective population sizes

\begin{tabular}{cll}
\hline $\begin{array}{l}\text { Effective population } \\
\text { size }\left(N_{\mathrm{e}}\right)\end{array}$ & Taxon & Reference \\
\hline $\begin{array}{c}\text { Long-term estimates } \\
102\end{array}$ & Galliformes birds & Barrowclough \& Shields, 1984 \\
106 & Non-passerine birds & Barrowclough \& Shields, 1984 \\
87 & Passerine birds & Barrowclough \& Shields, 1984 \\
$30-200$ & Mammals & Lande, 1979 \\
$40-300$ & Lower vertebrates & Lande, 1979 \\
$200-800$ & Drosophila & Lande, 1979 \\
5500 & American eel & Avise et al., 1988* \\
45000 & Catfish & Avise et al., 1988* \\
36700 & Red-winged blackbird & Avise et al., 1988* \\
$2250-4500$ & Man & Hartl, 1988 \\
Short-term estimates & & \\
$500-1000$ & D. pseudoobscura & Dobzhansky \& Wright, 1943 \\
315 & D. subobscura & Pollak, 1983 \\
400 & D. subobscura & Begon, 1977 \\
400 & Olive fruit fly & Pollak, 1983 \\
368 & Bufo marinus & Easteal, 1985 \\
4286 & Amerindian tribes & Chakraborty \& Neel, 1989 \\
\hline
\end{tabular}

*The studies by Avise et al. (1988) estimate female effective population size (these species were also chosen on the basis of their large census sizes and high rates of historical gene flow).

cients to be very large. Yet these estimates are much larger than those found in Table 7. If the estimates in Table 7 are correct, this level of selection cannot be reached with strict overdominance. If $N_{\mathrm{e}} \simeq 10^{3}$ then even an $N s$ as low as 100 implies $s \simeq 0.1$. This is very strong selection. Hence either the estimates of effective population sizes are incorrect or other forms of maintenance, such as frequency-dependent selection (with large advantages to rare genotypes), must be invoked.

One possible explanation, the effect of population subdivision, can probably be ruled out. All of the longterm estimates in Table 7 are explicitly estimates for the entire species and hence include the effects of population subdivision. In addition for the short-term estimates, unless migration is very rare, an estimate of local effective population size will equal that for the entire population. This is because $N_{\mathrm{e}}$ is the sum of the subpopulation sizes unless migration is very rare between some of the subpopulations (Ewens, 1979).

The demonstration of stasis at the molecular level requires strong and effective selection to act on polymorphisms for geological time periods. The magnitude of this selection places limits on the range of long-term effective population sizes and on the causative selective agent. If very long-term effective population sizes are less than a few thousand, then overdominance cannot explain shared polymorphisms. While frequencydependent selection can still explain shared polymorphisms with small effective sizes, it must provide very large advantages to alleles when they are rare. At the levels indicated here, selection is strong enough to act deterministically and effectively curtails random drift. This is reflected in Table 5; if the polymorphism is maintained, the allele frequencies seldom vary from their equilibrium point. This lack of variance may be a way to distinguish between overdominance and frequency dependence. These results also suggest that shared polymorphisms should often show identical allele frequencies within different species as has been found by Fisher et al. (1939) and Chiarelli (1963). The variance of allele frequency for these shared, polymorphic alleles should be examined.

\section{Acknowledgements}

I would like to thank B. Mongahan, N. Takahata, R. Chakraborty, J. Arnold and an anonymous reviewer for their comments on previous versions of a manuscript. This work was supported by Natural Sciences and Engineering Research Council grant number U0336. Additional support was provided by the Canadian Institute for Advanced Research. 


\section{References}

ARDEN, B. AND KLEIN, J. 1982. Biochemical comparison of major histocompatibility complex molecules from different subspecies of Mus musculus: evidence for transspecific evolution of alleles. Proc. Natl. Acad. Sci., USA, 79, 2342-2346.

ARDEN, B., WAKELAND, E. K. AND KLEIN, J. 1980. Structural comparisons of serologically indistinguishable $\mathrm{H}-2 \mathrm{~K}$ encoded antigens from inbred and wild mice. J. Immunol., 125, 2424-2438.

AVERY, P. J. 1978. Selection effects in a model of two intermigrating colonies of finite size. Theor. Pop. Biol., 13, 24-39.

AVISE, J. C., BALL, R. M. AND ARNOLD, J. 1988. Current versus historical population sizes in vertebrate species with high gene flow: A comparison based on mitochondrial DNA lineages and inbreeding theory for neutral mutations. $\mathrm{Mol}$. Biol. Evol., 5, 331-344.

BALNER, H., VANLEEWVEN, A., DERSJANT, H. AND VANROOD, J. 1967. Chimpanzee iso-antisera in relation to human leukocyte antigens. In: Curtoni, E. S., Matting, P. L. and Tosi, R. M. (eds) Histocompatibility Testing, 257-265 Copenhagen Munksgaard.

BARROWCLOUGH, G. F. AND SHIELDS, G. F. 1984. Karyotypic evolution and long-term effective population sizes of birds. The Auk, 101, 99-102.

BEGON, M. 1977. The effective size of a natural Drosophila subobscura population. Heredity, 38, 13-18.

CAVALLI-SFORZA, L. L. AND BODMER, W. F. 1971. The Genetics of Human Populations. Freeman \& Sons Publ. Co., San Francisco.

CHAKRABORTY, R. AND NEEL, J. v. 1989. Description and validation of a method for simultaneous estimation of effective population size and mutation rate from human population data. Proc. Natl. Acad. Sci., USA, 86, 9407-9411.

CHIARELL, B. 1963. Sensitivity to P.T.C. (phenyl-thiocarbamide) in primates. Folia Primatol., 1, 88-94.

DENNISTON, C. AND CROW, J. F. 1990. Alternative fitness models with the same allele frequency dynamics. Genetics, 125, 201-205.

DOBZHANSKY, T. AND WRIGHT, s. 1943. Genetics of natural populations. X. Dispersion rates of Drosophila pseudoobscura. Genetics, 28, 304-340.

EASTEAL, s. 1985. The ecological genetics of introduced populations of the giant toad Bufo marinus. II. Effective population size. Genetics, 110, 107-122.

EWENS, w. J. 1979. Mathematical Population Genetics. Springer-Verlag, New York.

FIGUEROA, F., GUNTHER, E. AND KLEIN, J. 1988. MHC polymorphism pre-dating speciation. Nature, 335, 265-267.

FISHER, R. A., FORD, E. B. AND HUXLEY, J. 1939. Taste-testing the Anthropoid Apes. Nature, 144, 750.

GILLESPIE, J. н. 1984. The molecular clock may be an episodic clock. Proc. Natl. Acad. Sci., USA, 81, 8009-8013.

GILLESPIE, J. H. 1986. Variability of evolutionary rates of DNA. Genetics, 113, 1077-1091.

GoulD, S. J. 1988. Prolonged stability in local populations of Cerion agassizi (Pleistocene-Recent) on Great Bahama Bank. Paleobiology, 14, 1-18.
GYLLENSTEN, U. B. AND ERLICH, H. A. 1989. Ancient roots for polymorphism at the HLA-DQ $\alpha$ locus in primates. Proc. Natl. Acad. Sci., USA, 86, 9986-9990.

haRTL, D. L. 1988. A Primer of Population Genetics. Sinauer Assoc. Inc., Sunderland, MA.

HEDrICK, P. w. 1983. Genetics of Populations. Science Books Int., Boston, MA.

JACQUARD, A. 1974. The Genetic Structure of Populations. Springer-Verlag, New York.

KIMURA, M. 1955. Solution of a process of random genetic drift with a continuous model. Proc. Natl. Acad. Sci., USA, 41, 144-150.

KIMURA, M. 1983. The Neutral Theory of Molecular Evolution. Cambridge University Press, New York.

KITCHIN, F. D. AND BEARN, A. G. 1965. The serum group specific component in non-human primates. Am. J. Hum. Genet., 17, 42-50.

KLEIN, J. 1980. Generation of diversity at MHC loci: implications for $\mathrm{T}$ cell receptor repertoires. Immunology, 80, 239-253.

LANDE, R. 1979. Effective deme sizes during long-term evolution estimated from rates of chromosomal rearrangement. Evolution, 33, 234-251.

LAWLOR, D.A., WARD, F. E., ENNIS, P. D., JACKSON, A. P. AND PARHAM, P. 1988. HLA-A and B polymorphisms predate the divergence of humans and chimpanzees. Nature, 335, 268-271.

MCCONNELL, T. J., TALBOT, W. S., MCINDOE, R. A. AND WAKELAND, E. K 1988. The origin of $\mathrm{MHC}$ class II gene polymorphism within the genus Mus. Nature, 332, 651-654.

MAYER, W. E., JONKER, M., KLEIN, D., IVANYI, P., VAN SEVENTER, G. AND KLEIN, J. 1988. Nucleotide sequences of chimpanzee MHC class I alleles: evidence for trans-species mode of evolution. $E M B O, 7,2765-2774$.

MOOR-JANKOWSKI, J., WIENER, A. S. AND ROGERS, C. M. 1964. Human blood group factors in non-human primates. Nature, 202, 663-665.

NEI, M. AND GRAUR, D. 1984. Extent of protein polymorphism and the neutral mutation theory. Evol. Biol., 17, 73-118.

oWEN, D. F. 1966. Polymorphism in pleistocene land snails. Science, 152, 71-72.

PARHAM, P., LAWLOR, D. A., LOMEN, C. E. AND ENNIS, P. D. 1989. Diversity and diversification of HLA-A,B,C alleles. $J$. Immunol., 142, 3937-3950.

POLLAK, E. 1983. A new method for estimating the effective population size from allele frequency changes. Genetics, 104, 531-548.

ROBERTSON, A. 1962. Selection for heterozygotes in small populations. Genetics, 47, 1291-1300.

SAGAI, T., SAKAIZUMI, M., MIYASHITA, N., BONHOMME, F., PETRAS, M. L., NIELSEN, J. T., SHIROISHI, T. AND MORIWAKI, K. 1989. New evidence for trans-species evolution of the H-2 class I polymorphism. Immunogenetics, 30, 89-98.

SPIESS, E. B. 1977. Genes in Populations. John Wiley \& Sons Inc., New York.

TAKAHATA, N. 1990. A simple genealogical structure of strongly balanced allelic lines and trans-species evolution of polymorphism. Proc. Natl. Acad. Sci., USA, 87, 2419-2423. 
TAKAHATA, N. AND NEI, M. 1990. Allelic genealogy under overdominant and frequency-dependent selection and polymorphism of major histocompatibility complex loci. Genetics, 124, 967-978.

WIENER, A. S. AND MOOR-JANKowSKI, J. 1963. Blood groups in anthropoid apes and baboons. Science, 142, 67-69.

wooD, J. w. 1987. The genetic demography of the Gainj of Papua New Guinea. II. Determinants of effective population size. Am. Nat., 129, 165-187.

WRIGHT, S. 1969. Evolution and the Genetics of Populations. Vol. II. Chicago University Press, Chicago.

\section{Appendix}

\section{Theory}

To derive the allele frequencies over time it is necessary to include the effects of mutation, drift and selection. Expanding the allele frequencies into a power series in $(N s)$ and ignoring higher powers of $(N s)$ offers an easy way to follow frequencies over time. This method of deriving expectations with selection acting in a finite population was first illustrated by Kimura (1955) and again by Avery (1978). The expectation $E^{t}(x)$ is described as a linear function of expectations evaluated at $N s=0\left(s_{1}=s_{2}=s\right)$. Consider a population that has only small changes in allele frequency $x$ from one generation to the next. If this small change is designated as $\delta x$, then the expected $n$th power of the allele frequency in the next generation is

$$
\begin{aligned}
E\left[(x+\delta x)^{n}\right]= & E\left(x^{n}\right)+n E\left[\left(x^{n-1} E(\delta x \mid x)\right]\right. \\
& +n(n-1) / 2 E\left[x^{n-2} E\left(\delta x^{2} \mid x\right)\right]+\ldots
\end{aligned}
$$

For the above model,

$$
E(\delta x \mid x)=\mu(1-2 x)+s x\left(1-3 x+2 x^{2}\right)
$$

and

$$
E\left(\delta x^{2} \mid x\right)=x(1-x) / 2 N \text {. }
$$

Substituting [A2-3] into [A1] yields,

$$
\begin{aligned}
& E(x+\delta x)=\mu+(1-2 \mu+s) E(x)-3 s E\left(x^{2}\right)+2 s E\left(x^{3}\right) \\
& E\left[(x+\delta x)^{2}\right]=(2 \mu+1 / 2 N) E(\mathrm{x})
\end{aligned}
$$

$$
\mathbf{M}=\left[\begin{array}{ccc}
1-2 \mu, & 0, & 0, \\
2 \mu+1 / 2 N, & 1-4 \mu-1 / 2 N, & 0, \\
0, & 3 \mu+3 / 2 N, & 1-6 \mu-3 / 2 N, \\
0, & 0, & 4 \mu+6 / 2 N, \\
s, & -3 s, & 2 s, \\
0, & 2 s, & -6 s,
\end{array}\right.
$$

$$
\begin{aligned}
& +(1-4 \mu-1 / 2 N+2 s) E\left(x^{2}\right) \\
& -6 s E\left(x^{3}\right)+4 s E\left(x^{4}\right) .
\end{aligned}
$$

A similar equation for the change in $E\left(x^{3}\right)$ with selection requires that still higher order moments be determined. Unless $s=0$, this leads to an infinite system of equations describing all allele frequency moments. It is possible, however, to determine a series solution for $E(x)$ in powers of $(N s)$. For example, consider $E\left(x^{n}\right)$ expanded into a series in $\mathrm{Ns}$

$$
E\left(x^{n}\right)=a+b(N s)+c(N s)^{2}+d(N s)^{3}+\ldots
$$

Obviously $a=\left.E\left(x^{n}\right)\right|_{N_{s}=0}$ but $b, c$ and $d$ are unknowns. $E(x)$ can be found as a linear function of $N s(c=d=0)$ by determining the value of the unknown $b$. This can be found by substituting [A5] into [A4] and ignoring terms higher than $(N s)$. Hence,

$$
\begin{aligned}
E(x+\delta x)=\mu & +(1-2 \mu) E(x)+\left.s E(x)\right|_{s=0} \\
- & \left.3 s E\left(x^{2}\right)\right|_{s=0}+\left.2 s E\left(x^{3}\right)\right|_{s=0} \\
E\left[(x+\delta x)^{2}\right]= & (2 \mu+1 / 2 N) E(x) \\
& +(1-4 \mu-1 / 2 N) E\left(x^{2}\right)+\left.2 s E\left(x^{2}\right)\right|_{s=0} \\
& -\left.6 s E\left(x^{3}\right)\right|_{s=0}+\left.4 s E\left(x^{4}\right)\right|_{s=0} .
\end{aligned}
$$

Equations [A6] describe a finite system of equations that determine $E(x)$ and $E\left(x^{2}\right)$ to terms of the order $(N s)$ but ignores terms of the order $(N s)^{2}$ and higher. This system gives the expected change in allele frequency in one generation. The expression for $t$ generations must be found by iterating these equations. This iteration must include all terms in $t$ and $s$ so that nothing is ignored. Since this is a linear system of equations it can be solved exactly using standard methods. This can be most easily done by rewriting the equations in matrix notation.

Including the recursion equation for the expectations when $s=0$, the equations [A6] can be rewritten as

$\mathbf{Z}^{t+1}=\mathbf{M Z}^{t}+\mathbf{V}$

where

$$
\begin{aligned}
\mathbf{Z}= & {\left[\left.E^{t}(x)\right|_{s=0},\left.E^{t}\left(x^{2}\right)\right|_{s=0},\left.E^{t}\left(x^{3}\right)\right|_{s=0},\left.E^{t}\left(x^{4}\right)\right|_{s=0},\right.} \\
& \left.E^{t}(x), E^{t}\left(x^{2}\right)\right]^{T} \\
\mathbf{V}= & {[\mu, 0,0,0, \mu, 0]^{T} }
\end{aligned}
$$

$$
\left.\begin{array}{ccc}
0, & 0, & 0 \\
0, & 0, & 0 \\
0, & 0, & 0 \\
1-8 \mu-6 / 2 N, & 0, & 0 \\
0, & 1-2 \mu, & 0 \\
4 s, & 2 \mu+1 / 2 N, & 1-4 \mu-1 / 2 N
\end{array}\right]
$$


To solve this system note that $\mathbf{M}$ can be decomposed into $\mathbf{P D P}^{-1}$ where

$\mathbf{D}=\left[\begin{array}{cccccc}1-2 \mu, & 0, & 0, & 0, & 0, & 0 \\ 0, & 1-4 \mu-1 / 2 N, & 0, & 0, & 0, & 0 \\ 0, & 0, & 1-6 \mu-3 / 2 N, & 0, & 0, & 0 \\ 0, & 0, & 0, & 1-8 \mu-6 / 2 N, & 0, & 0 \\ 1, & 0, & 0, & 0, & 1-2 \mu, & 0 \\ 0, & 1, & 0, & 0, & 0, & 1-4 \mu-1 / 2 N\end{array}\right]$

With $\theta=4 N \mu, \mathbf{P}$ is

$\left[\begin{array}{cccccc}-(3+2 \theta) / \theta s, & 0, & 0, & 0, & 0, & 0 \\ -(3+2 \theta) / \theta s, & -(5+2 \theta) / s(2 \theta-1), & 0, & 0, & 0, & 0 \\ -(6+3 \theta) / 2 \theta s, & -(15+6 \theta) / 2 s(2 \theta-1), & -(3+2 \theta) / 4 N s, & 0, & 0, & 0 \\ -(3+\theta) / \theta s, & -(9+3 \theta) / s(2 \theta-1), & -(3+2 \theta) / 2 N s, & -(5+2 \theta) / 8 N s, & 0, & 0 \\ 1, & 0, & 1, & 0, & 1, & 0 \\ 1, & 1, & 1, & 1, & 1, & 1\end{array}\right]$

and $\mathbf{P}^{-1}$ is

$\left[\begin{array}{cccccc}\frac{-s \theta}{(3+2 \theta)^{\prime}}, & 0, & 0, & 0, & 0, & 0 \\ \frac{s(2 \theta-1)}{(5+2 \theta)}, & \frac{-s(2 \theta-1)}{(5+2 \theta)}, & 0, & 0, & 0, & 0 \\ \frac{-6 N s(1+\theta)}{(3+2 \theta)^{2}}, & \frac{6 N s}{(3+2 \theta)^{\prime}} & \frac{-4 N s}{(3+2 \theta)^{\prime}} & 0, & 0, & 0 \\ \frac{8 N s(1+\theta)}{(5+2 \theta)^{2}}, & \frac{-24 N s(2+\theta)}{(5+2 \theta)^{2}}, & \frac{16 N s}{(5+2 \theta)}, & \frac{-8 N s}{(5+2 \theta)}, & 0, & 0 \\ \frac{s \theta}{(3+2 \theta)}+2 N s \frac{3+3 \theta}{(3+2 \theta)^{2}}, & \frac{-6 N s}{(3+2 \theta)^{\prime}} & \frac{4 N s}{(3+2 \theta)^{\prime}}, & 0, & 1, & 0 \\ -s \frac{2 \theta-1}{(5+2 \theta)}-2 N s \frac{4+4 \theta}{(5+2 \theta)^{2}}, & s \frac{2 \theta-1}{(5+2 \theta)}+2 N s \frac{24+12 \theta}{(5+2 \theta)^{2}}, & \frac{-16 N s}{(5+2 \theta)}, & \frac{8 N s}{(5+2 \theta)^{\prime}}, & -1, & 1\end{array}\right]$

The transient solution is then given by

$\mathbf{Z}^{t}=\mathbf{P D}^{t} \mathbf{P}^{-1} \mathbf{Z}^{0}+\sum_{i=0}^{t-1} \mathbf{P D}^{\mathrm{i}} \mathbf{P}^{-1} \mathbf{V}$

and the sum is easily solved due to the nature of $\mathbf{D}$. Note that higher allele frequency moments can be easily found. Furthermore, the linear approximations can be used to find quadratic approximations and so on to any desired degree of accuracy.

The expected heterozygosity can be found as

$H e t=\mathrm{E}^{\mathrm{t}}\left(x^{2}\right)+E^{t}\left[(1-x)^{2}\right]$.
Solutions to this equation are shown in Table A1. The solutions to these equations agree with the simulation results. Both demonstrate that for neutral alleles the expected heterozygosity will be very small after $4 N_{\mathrm{e}}$ or more generations. The strongest selection permitted by this linear solution is $4 N s=0.1$. Even at this level of selection, however, the theoretical solutions and the simulations in Table A1 again demonstrate that polymorphism cannot be maintained. A theoretical approach that approximates larger selection coefficients has been pioneered by Takahata (1990). 
Table A1 Expected heterozygosity within a finite population

\begin{tabular}{|c|c|c|c|c|}
\hline & $N_{\mathrm{e}}=10^{6}$ & $N_{\mathrm{e}}=10^{5}$ & $N_{\mathrm{e}}=10^{4}$ & $N_{\mathrm{e}}=10^{3}$ \\
\hline \multicolumn{5}{|c|}{ a) Theoretical results, $4 \mathrm{Ns}$} \\
\hline$t=10^{5}$ & 0.476 & 0.303 & $3.41 \times 10^{-3}$ & $4.00 \times 10^{-6}$ \\
\hline$t=10^{6}$ & 0.304 & $3.75 \times 10^{-3}$ & $4.00 \times 10^{-5}$ & $4.00 \times 10^{-6}$ \\
\hline$t=10^{7}$ & $7.18 \times 10^{-3}$ & $4.00 \times 10^{-4}$ & $4.00 \times 10^{-5}$ & $4.00 \times 10^{-6}$ \\
\hline$t=10^{8}$ & $3.97 \times 10^{-3}$ & $4.00 \times 10^{-4}$ & $4.00 \times 10^{-5}$ & $4.00 \times 10^{-6}$ \\
\hline
\end{tabular}

(b) Simulation results, $4 \mathrm{Ns}=0$

$\begin{array}{llccl}t=10^{5} & 0.480 \pm 0.002 & 0.304 \pm 0.013 & 0.008 \pm 0.004 & 0.0 \pm 0.0 \\ t=10^{6} & 0.300 \pm 0.013 & 0.000 \pm 0.000 & 0.0 \pm 0.0 & - \\ t=10^{7} & 0.011 \pm 0.005 & 0.0 \pm 0.0 & - & - \\ t=10^{8} & 0.002 \pm 0.002 & - & - & -\end{array}$

(c) Theoretical results, $4 N s=0.1$

$\begin{array}{lllll}t=10^{5} & 0.476 & 0.304 & 3.57 \times 10^{-3} & 4.03 \times 10^{-6} \\ t=10^{6} & 0.305 & 3.92 \times 10^{-3} & 4.07 \times 10^{-5} & 4.03 \times 10^{-6} \\ t=10^{7} & 7.40 \times 10^{-3} & 4.06 \times 10^{-4} & 4.07 \times 10^{-5} & 4.03 \times 10^{-6} \\ t=10^{8} & 4.03 \times 10^{-3} & 4.06 \times 10^{-4} & 4.07 \times 10^{-5} & 4.03 \times 10^{-6}\end{array}$

(d) Simulation results, $4 N s=0.1$

\begin{tabular}{lllll}
$t=10^{5}$ & $0.478 \pm 0.002$ & $0.301 \pm 0.013$ & $0.005 \pm 0.003$ & $0.0 \pm 0.0$ \\
$t=10^{6}$ & $0.311 \pm 0.013$ & $0.000 \pm 0.000$ & $0.0 \pm 0.0$ & - \\
$t=10^{7}$ & $0.001 \pm 0.001$ & $0.000 \pm 0.000$ & - & - \\
$t=10^{8}$ & $0.002 \pm 0.001$ & - & - & - \\
\hline
\end{tabular}

This table gives the expected heterozygosity within one population for neutral or selected alleles (overdominant selection with $4 N s=0.1$ ). Simulations were repeated 200 times to calculate the mean and its standard error. Simulations used an effective population size of $N=100$ diploid individuals $(2 N=200)$. The mutation rate was adjusted to an amount equivalent to $\mu=10^{-9}$. 